\title{
Cultural adaptation and Northern Sotho translation of the Modified Checklist for Autism in Toddlers
}

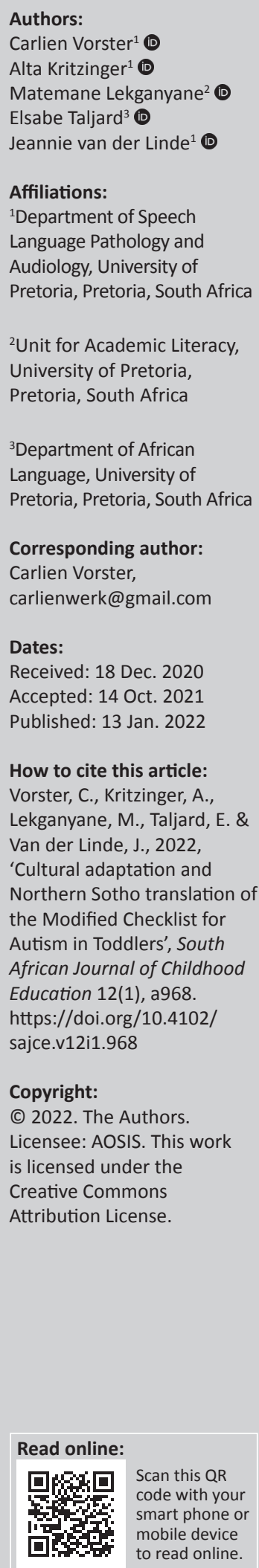

Background: In recent reviews of autism spectrum disorder screening tools, the Modified Checklist for Autism in Toddlers, Revised with Follow-Up (M-CHAT-R/F ${ }^{\mathrm{TM}}$ ) has been recommended for use in lower middle-income countries to promote earlier identification.

Aim: The study aim was to culturally adapt and translate the M-CHAT-R/F ${ }^{\mathrm{TM}}$ into Northern Sotho, a South African language.

Setting: An expert panel was purposively selected for the review and focus group discussion that was conducted within an academic context.

Method: The source translation (English) was reviewed by bilingual Northern Sotho-English speech-language therapists who made recommendations for cultural adaptation. A double translation method was used, followed by a multidisciplinary expert panel discussion and a self-completed questionnaire.

Results: Holistic review of test, additional remarks and grammar and phrasing were identified as the most prominent themes of the panel discussion, emphasising the equivalence of the target translation.

Conclusion: A South African culturally adapted English version of the M-CHAT-R/F ${ }^{\mathrm{TM}}$ is now available along with the preliminary Northern Sotho version of the M-CHAT-R/F ${ }^{\mathrm{TM}}$. The two versions can now be confirmed by gathering empirical evidence of reliability and validity.

Keywords: autism screening test; cultural adaptation; test translation; Northern Sotho M-CHAT-R/FTM; South Africa; double translation method; test equivalence.

\section{Introduction}

Autism spectrum disorder (ASD) is universally recognised as a rising public health concern (World Health Organization 2013:6), yet almost all knowledge gained about the condition stems from high-income countries (HICs). In a scoping review of ASD research in sub-Saharan Africa (SSA), it was found that studies published prior to October 2015 mainly targeted communicable diseases, with a limited focus on neurodevelopmental disorders like ASD (Franz et al. 2017:723). The latest report of ASD diagnosis in the United States (US) indicates a rising trend from 1 in 69 children in 2012 to 1 in 54 in 2020 (Centres for Disease Control 2012; Hyman et al. 2020:2). As the extent of ASD in SSA is not well known, the need for early identification programmes is amplified.

The World Health Organization (2005) considers early identification as a high priority, as detection at a young age may decrease the impact of impairments. Awareness and early identification of ASD in infancy are vital to improve long-term outcomes and optimise opportunities for children with ASD to benefit from early intensive intervention (Hyman et al. 2020:1; Zwaigenbaum et al. 2015:s10). Despite international efforts to increase awareness of ASD using screening, large-scale studies conducted in the US still indicate that the mean age of diagnosis remains at $4-5$ years of age and even later in lower socio-economic communities because of the heterogeneity of symptoms (Centers for Disease Control and Prevention 2014:1; Hyman et al. 2020:2; Robins et al. 2014:s11). Early identification and intervention for young children with ASD therefore remain a challenge that requires a global effort, with lower middle-income countries (LMICs) actively contributing.

While screening tools are well established in HICs (Marlow, Servili \& Tomlinson 2019:177), research from LMICs highlights the need for culturally appropriate ASD screening and diagnostic tools. Standardised screening tools from HICs have been validated for their particular settings, but the use of these tools in other cultures or LMICs are often associated with significant limitations

Note: Northern Sotho is also referred to as Sepedi or Sesotho sa Leboa. The correct designation for this language is an ongoing debate of which the authors are aware. 
in score interpretation and feasibility (Abubakar et al. 2008:217; Fisher, Morris \& Martines 2014:314; Hyman et al. 2020:12). The interpretation of screening tools is complicated when used in different contexts because the content, reliability and validity have been developed for a specific population and language (Rahman, Waheed \& Hussain 2003:1). The feasibility of the tool in another setting is influenced by aspects such as lack of clinician and parental knowledge, limited time for orientation, different cultural perceptions and restricted human resources and finance (Kautzky \& Tollman 2008:24; Morelli et al. 2014:9; Soto et al. 2015; Stewart \& Lee 2017:528; Van der Merwe et al. 2017:1). A possible solution for barriers posed is the development of a contextually relevant caregiver-administered screening test, by means of adaptation and translation, to identify ASD across different socio-economic backgrounds and cultures without requiring extensive resources (Fyvie et al. 2016:417; Rahman et al. 2003:1).

The Modified Checklist for Autism in Toddlers, Revised, with Follow-Up (M-CHAT-R/ $\mathrm{F}^{\mathrm{TM}}$ ) is deemed an appropriate caregiver-administered screening test for the LMIC context and is already translated into 56 languages (Marlow et al. 2019:189; M-CHAT ${ }^{\text {TM }}$ website, accessed 13 March 2020). The recommendation for use is based on reviewing criteria such as the sensitivity and specificity of data, the sample size of the validation study, the cost to conduct the tool, the use in LMICs and whether the instrument can be used by community health workers (CHWs). The M-CHAT-R/F ${ }^{\mathrm{TM}}$ showed high sensitivity (91\%) and specificity (95\%) (Robins et al. 2014:41). In addition, the tool was standardised with a sample of more than 300 toddlers, is free of charge and adapted versions are already used in countries classified as LMICs (Marlow et al. 2019:188). With the revision of the $\mathrm{M}-\mathrm{CHAT}-\mathrm{R} / \mathrm{F}^{\mathrm{TM}}$ in 2014, meaningful reduction in the screenpositive rate (an increased risk of having ASD) and an increase in the detection of ASD in the US were observed (Robins et al. 2014:37).

Recent research showed that children with ASD are still being overlooked with the use of the English version of the instrument (Beacham et al. 2018:687; Guthrie et al. 2019:8). It appears that children from lower income households, who receive public healthcare and have been exposed to a language other than English, are being overlooked or incorrectly identified as at risk when the English version of the M-CHAT-R/F $\mathrm{F}^{\mathrm{TM}}$ is used (Guthrie et al. 2019). This oversight may result in lower sensitivity and specificity. Lower specificity may be attributed to unfamiliarity with the cultural and linguistic concepts used in the M-CHAT-R/F ${ }^{\mathrm{TM}}$, providing a rationale for the adaptation and translation of this test. To limit the variance in early diagnosis because of cultural differences, adaptation and validation measures are necessary (Hyman et al. 2020:8).

While the development of new instruments is expensive and time-consuming, most health constructs are universal, thus allowing application in various populations after cultural adaptation and if necessary, translation (Rahman et al. 2003:1).
The M-CHAT-R/F $\mathrm{F}^{\mathrm{TM}}$ is already available in many official translations of the English version, but none of the translations includes any of the indigenous languages of South Africa (M-CHAT ${ }^{\mathrm{TM}}$ website, accessed 13 March 2020). Northern Sotho is the third most spoken indigenous language in South Africa, with $12 \%$ of the population communicating in the language (StatsSA 2019). The language is most commonly spoken in the northern provinces of South Africa, with $42 \%$ of the residents of Mamelodi, a township within the City of Tshwane Metropolitan Municipality, using Northern Sotho. The aim of the study was thus to culturally adapt and translate the M-CHAT-R/FTM and its test instructions into Northern Sotho. This adaptation and translation aimed to ensure an accurate version that reads fluently and appears authentic in the target language.

\section{Method}

\section{Research design and ethical considerations}

A qualitative design was used so that perspectives, opinions and suggestions of experts could be reflected. Data were derived from self-completed questionnaires that included rating scales and structured review questions adapted from the International Test Commission [ITC](ITC 2016). Following the ITC and World Health Organization (2019) guidelines, a test adaptation and double translation method were adopted. This method aimed to avoid a literal translation by rather ensuring the similar meaning of constructs (equivalence) across the two languages (ITC 2016). The source translation was adapted to remove cultural biases and improve relevance before translating the test into the target language followed by a back translation. A comparative approach where the source, target and back translations were compared for functional, conceptual and linguistic equivalence was followed (ITC 2016). This implied that the study was conducted in three consecutive phases: the adaptation, translation and reconciliation. Permission to use and translate the tool was given by information redacted to maintain the integrity of the review process.

\section{Participants}

Panel members involved in the different phases of the study were specialists from diverse multidisciplinary fields as required by the task they had to perform (see Table 1). The purpose was to draw on diverse specialist opinions, thereby following a comprehensive approach to test adaptation and translation. Two professional translators ensured the linguistic and conceptual equivalence of the target and back translations to prevent literal and direct translation. An educational psychologist, Northern Sotho linguists, speechlanguage therapist (SLT) practitioners with clinical experience working with parents and children with ASD, as well as researchers with expertise in instrument development and translation were included. Four of the nine panel members had in-depth knowledge of Northern Sotho culture to identify references that will be misunderstood in Northern Sotho. 
TABLE 1: Participants' expertise and role.

\begin{tabular}{|c|c|c|c|c|}
\hline Participant code & Participant description & Highest qualification & Field of expertise & Role \\
\hline P1 & Academic expert & $\mathrm{PhD}$ & $\begin{array}{l}\text { Multilingual speaker proficient in Afrikaans, English } \\
\text { and Northern Sotho. } \\
\text { Northern Sotho linguist }\end{array}$ & $\begin{array}{l}\text { Member of the expert panel: Review of Grammar } \\
\text { and phrasing, and Passage translation }\end{array}$ \\
\hline P3 & SLT & $\begin{array}{l}\text { M. Communication } \\
\text { Pathology: Speech- } \\
\text { Language Pathology }\end{array}$ & Bilingual Northern Sotho-English SLT. & Cultural adaptation \\
\hline P4 & SLT & $\begin{array}{l}\text { B. Speech and Hearing } \\
\text { Therapy }\end{array}$ & Bilingual Northern Sotho-English SLT. & $\begin{array}{l}\text { Cultural adaptation } \\
\text { Member of the expert panel: Review of General, } \\
\text { Item format, Culture }\end{array}$ \\
\hline P5 & Educational psychologist & $\mathrm{PhD}$ & $\begin{array}{l}\text { Specialist in childhood development and } \\
\text { psychometry. Bilingual Afrikaans-English speaker. }\end{array}$ & $\begin{array}{l}\text { Member of the expert panel: Review of General, } \\
\text { Item format, Culture }\end{array}$ \\
\hline P6 & Second translator & Language Practitioner & $\begin{array}{l}\text { Professional translator. } \\
\text { Bilingual Northern Sotho-English speaker. }\end{array}$ & Back translation \\
\hline R1 & Researcher & $\mathrm{PhD}$ & $\begin{array}{l}\text { SLT and ASD specialist. } \\
\text { M-CHAT-R/F user. } \\
\text { Bilingual Afrikaans-English speaker. }\end{array}$ & $\begin{array}{l}\text { Member of the expert panel: Review of Grammar } \\
\text { and phrasing, and Passage translation }\end{array}$ \\
\hline R2 & Researcher & $\mathrm{PhD}$ & $\begin{array}{l}\text { SLT, specialist in tool development and translation. } \\
\text { Bilingual Afrikaans-English speaker. }\end{array}$ & Member of the expert panel \\
\hline R3 & Researcher & $\mathrm{PhD}$ & $\begin{array}{l}\text { Professional translator. } \\
\text { Multilingual speaker proficient in Northern Sotho, } \\
\text { Setswana, English and Afrikaans. }\end{array}$ & $\begin{array}{l}\text { Translation of source text into Northern Sotho } \\
\text { Member of the expert panel }\end{array}$ \\
\hline
\end{tabular}

SLT, speech-language therapist; ASD, autism spectrum disorder; M-CHAT-R/F, Modified Checklist for Autism in Toddlers, Revised with Follow-Up.

\section{Material}

The English M-CHAT-R/FTM (Robins, Fein \& Barton 2018) was the source translation used in the study. The electronic or hard-copy-administered screening test consists of two sections (20 yes or no questions with related child behavioural examples and follow-up questions), completed by caregivers with a minimum Grade 4 level education. The test was developed to screen for risk of ASD-specific traits in children aged 16-30-months. For all 20 questions except items 2, 5 and 12 , 'No' indicates a risk for ASD. A child's total score is categorised according to low-risk (zero to two), medium-risk (three to seven) and high-risk (8-20) categories for ASD. Medium-risk classification requires monitoring the child and completing the follow-up section. The section is presented in a user-friendly flowchart format with questions and different behavioural examples from the first section, indicating PASS/FAIL. When a toddler fails two or more of these questions, a referral for a diagnostic evaluation is recommended. High-risk requires immediate referral to a specialist doctor. The design of the follow-up section allows the parent to answer the same questions as the initial 20 questions, but with different child behaviour examples. By offering different examples in the two sections, more opportunities for clarification of behaviour are provided, preventing risk identification based on one example only. This feature contributes positively to the validity of the test, resulting in less false positive cases. If the toddler is still identified as being at risk after completion of the follow-up questions, a referral to a professional is required.

The second tool, the Item Translation and Adaptation Review Questionnaire (Hambleton \& Zenisky 2011:71), was used in the reconciliation phase of the study. The in-depth review questionnaire includes five categories, General, Item format, Culture, Grammar and phrasing, and Passage translation, judged according to a four-point Likert scale (Yes, No, Unsure and Not Relevant). Specialists were requested to complete the questionnaire based on the different translations, prior to the panel discussion. Selected panel members (P1, P2, P4, P5, R2) were requested to complete the questionnaire a second time, following additional changes during the reconciliation phase.

\section{Procedures and data collection}

According to the Pre-condition Guidelines (ITC 2016) of the adaptation phase, permission to use the M-CHAT-R/F $\mathrm{F}^{\mathrm{TM}}$ was obtained, then the Northern Sotho-English SLTs identified the possible linguistic and cultural incompatibilities of the test. After careful consideration of the source translation, P3 and P4 answered two questions: 'Which test items and instructions in the English version are culturally specific, and may not be applicable to Northern Sotho?' and 'Which concepts or words will be difficult to translate to Northern Sotho?' P3 and P4 were also requested to propose culturally appropriate solutions or adaptations that were discussed with R3.

The next phase entailed the translation of the adapted test aiming for conceptual equivalence between the two translations. P6, blind to the original English version of the $\mathrm{M}-\mathrm{CHAT}-\mathrm{R} / \mathrm{F}^{\mathrm{TM}}$, conducted the back translation into English. The aim of the back translation was to allow the expert panel to double-check the target version and to reconcile the two translations while maintaining content and construct equivalence (ITC 2016:13). The back translation thus served as a measure to evaluate the accuracy of the target translation for panel members who were not Northern Sotho speakers and was discarded afterwards.

During the reconciliation phase, the expert panel met for a 90-min recorded discussion of the translations, facilitated by the principal researcher. The panel members studied the translations and completed the Item Translation and Adaptation Review Questionnaire prior to the discussion. As all participants were not Northern Sotho speakers, only three members $(\mathrm{P} 1, \mathrm{P} 2, \mathrm{P} 4)$ of the panel compared the source translation as 
TABLE 2: Cultural adaptations to the Modified Checklist for Autism in Toddlers, Revised with Follow-Up.

\begin{tabular}{|c|c|c|c|c|}
\hline Question & P3 (SLT) & P4 (SLT) & Adaptations & Justification \\
\hline $\begin{array}{l}\text { 'Which test items and } \\
\text { instructions in the English } \\
\text { version are culturally specific, } \\
\text { and may not be applicable to } \\
\text { Northern Sotho?' }\end{array}$ & $\begin{array}{l}\text { 'Does your } \\
\text { child look } \\
\text { you in the } \\
\text { eye' }\end{array}$ & 'Eye contact' & $\begin{array}{l}\text { 'Does your child look in your direction or in the } \\
\text { eye when you are talking to them?' }\end{array}$ & $\begin{array}{l}\text { In Northern Sotho culture, it is culturally inappropriate } \\
\text { for children to make eye contact with older or superior } \\
\text { people when talking to them. }\end{array}$ \\
\hline \multirow{2}{*}{$\begin{array}{l}\text { 'Which concepts or words } \\
\text { will be difficult to translate } \\
\text { into Northern Sotho?' }\end{array}$} & $\begin{array}{l}\text { 'Pretend } \\
\text { play' }\end{array}$ & 'Soft toy' & \multirow{2}{*}{$\begin{array}{l}\text { 'Pretend play' was regarded as synonymous to } \\
\text { 'Make-believe' and was adapted to 'acting'. } \\
\text { 'Soft toy' was simplified to 'toy', } \\
\text { 'Playground equipment' was changed to 'tree'. }\end{array}$} & \multirow{2}{*}{$\begin{array}{l}\text { Both 'soft toys' and 'playground equipment' are } \\
\text { culturally different and limited access may affect } \\
\text { comprehension as children from disadvantaged } \\
\text { communities may not have access to outdoor Jungle } \\
\text { Gym equipment. }\end{array}$} \\
\hline & 'Equipment' & 'Playground equipment' & & \\
\hline
\end{tabular}

SLT, speech-language therapist.

well as target translation and back translation. The other panel members reviewed the target translation and back translation or the source translation and back translation. This allowed the panel to critically evaluate the linguistic and cultural equivalence between the Northern Sotho and source translation. Recommendations were considered and the best solutions were identified during a critical discussion. The linguists (P1 \& P2) identified linguistically correct options and first language Northern Sotho speakers (P4 \& R3) confirmed the local use of the vocabulary. A verbatim transcription of the voice recording of the panel discussion was analysed.

\section{Data analysis}

Qualitative data of the cultural adaptation were analysed according to the contribution of each participant. Data were classified, coded, tabulated and summarised according to specific constructs (Schurink, Fouché \& De Vos 2015:402; Sutton \& Austin 2015:227).

The panel discussion data were analysed in three cycles, including both manual and electronic coding, thereby providing a more trustworthy account (Saldaña 2016:27). During the preliminary analysis, cycle data were clustered into the five main categories of the Item Translation and Adaptation Review Questionnaire (Hambleton \& Zelenski 2011:71). The second cycle involved 'splittering' separating content into smaller codes. 'Splittering' implied that more detailed descriptive codes were assigned to the content of the five categories to identify specific errors and incompatibilities indicated during the panel discussion. The Computerassisted qualitative data analysis software (CAQDAS) software (ATLAS.ti Version 8) was used for splittering. The third cycle involved the reconfiguration of codes into themes. Detailed codes were 'lumpered' (grouped together) according to five main themes, to determine which theme emerged as most prominent during the panel discussion (Saldaña 2016).

\section{Results}

\section{Adaptation phase}

Table 2 depicts the results of the questions posed to the two Northern Sotho SLTs and the adaptations made to the source translation based on the proposed suggestions.
There was agreement between the two SLTs (P3 and 4) that a child making eye contact when communicating with an elder is culturally specific and not typically observed in Northern Sotho culture. This non-verbal communication custom is also evident in other Southern African cultures, as it is considered a sign of respect when a minor refrains from making eye contact with a superior (Mncwango 2009:51). The construct of 'make-believe' or 'pretend play' was also identified by both SLTs as not applicable in Northern Sotho culture and a concept that will be difficult to translate. It appears that the concept of 'pretend play' does not feature within Northern Sotho culture. 'Playground equipment' was identified as an unfamiliar concept in disadvantaged communities in South Africa. As the aim of a test translation is to achieve maximum comprehension by its users while still maintaining conceptual equivalence, it was important to find acceptable solutions.

\section{Translation phase}

The adapted English version of the test was used as source translation. The forward translation to Northern Sotho was completed within 14 days. P6 performed the back translation while blinded to the source translation. The translation phase therefore produced the target translation and back translation.

\section{Reconciliation phase}

Figure 1 depicts the broad outline of the three cycles and corresponding codes and themes that were identified during analysis of the 90-min-long panel discussion recording.

The category Item format initially included in the preliminary cycle was omitted. The original user-friendly organisation and item format of the M-CHAT-R/FTM were retained. Four of the initial five categories were identified as themes with Additional remarks replacing Item format as the fifth theme. The identification of an additional nine detailed codes, not relating to the other categories, is evidence of the scope and comprehensiveness of the panel discussion.

The most often discussed detailed codes are under the theme Holistic review of test. Five detailed codes (discussed 106 times) were 'splittered' under the theme. The detailed code occurring most frequently was the confirmation that content equivalence was achieved between the different test items in the source translation, target translation and back translation. 
Facilitator: The example said 'pointing to a snack or a toy' which was back translated to 'point to a light food'?

P2: It is a snack in Northern Sotho.

The following example indicates that construct and linguistic equivalence between the source translation, target translation and back translation were achieved, even though the vocabulary differs.

Facilitator: Does your child try to copy you? The back translation says 'Does your child imitate or copy what you are doing...'

The panel agreed that the linguistic meaning was preserved between the three different translations.
Twelve of the 20 test items of the source M-CHAT-R/F ${ }^{\mathrm{TM}}$ version were accurately translated in the target translation. An example of the equivalence of the double translation being:

Source translation item-nr 2: Have you ever wondered if your child might be deaf?

Target translation: Naa o ile wa nagana gore ngwana wa gago e ka ba e le sefoa?

Back translation: Have you ever wondered/think if your child is deaf?

Recommendations were made when there was a lack of linguistic $(n=19)$ and construct $(n=5)$ equivalence between the

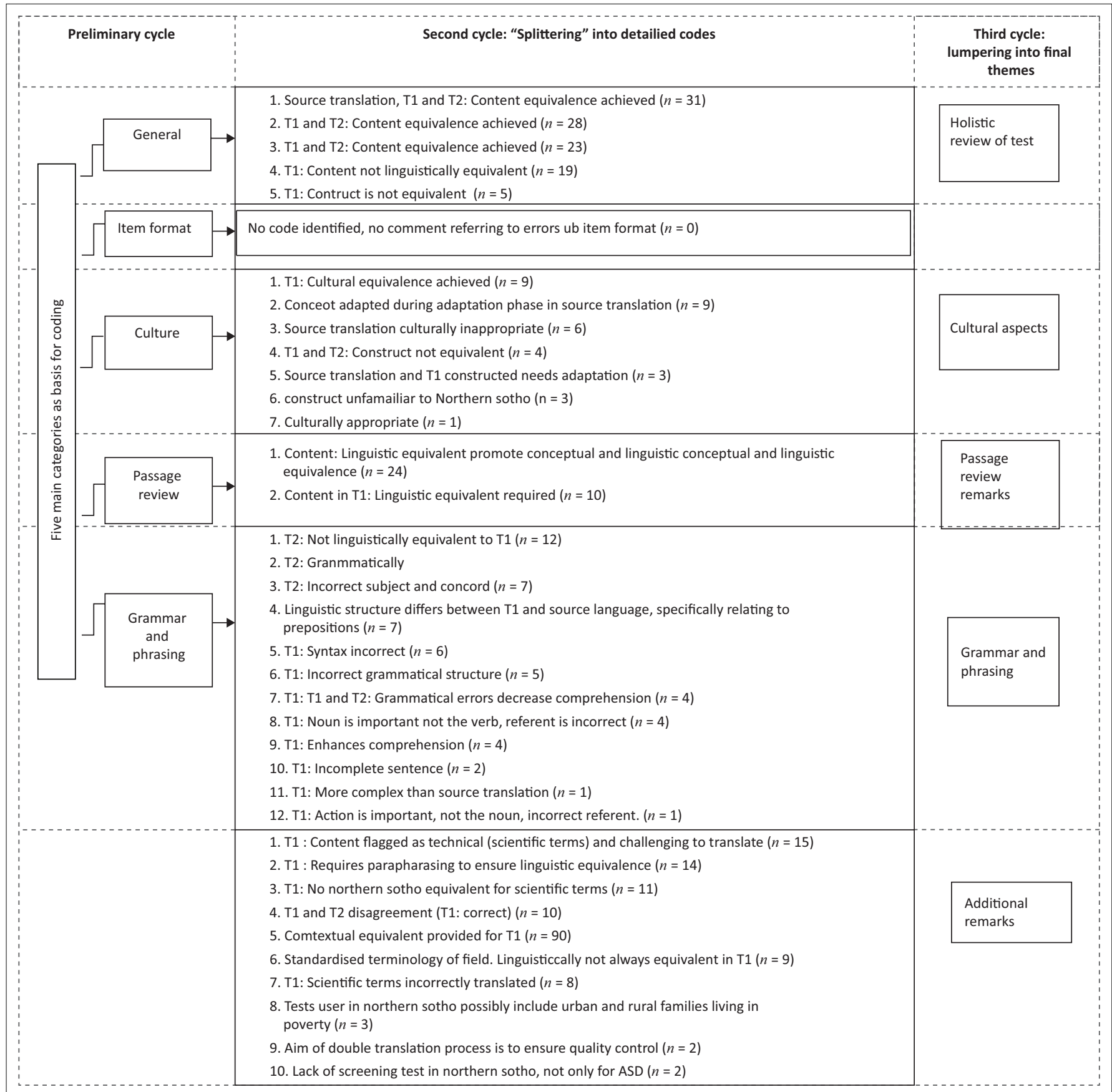

ASD, autism spectrum disorder.

*, T1: target translation, T2: back translation.

FIGURE 1: Cycles analysis and identification of codes and themes. 
target translation and the source translation. With the combined knowledge of the expert panel, most of the translation problems were therefore solved during the discussion.

The second most discussed theme was 10 detailed codes under Additional remarks (Theme 5). These codes related to and clarified instances where linguistic equivalence was not achieved in Theme 1. The most prominent code referred to the content that was flagged as technical and scientific terms that were challenging to translate $(n=15)$ :

P2: I think this is a difficult thing to translate because of the technical nature.

R2: If I can just say that the technical stuff is something that we have to make sure that we find solutions for because those...

P2: I think it is a very technical one and if you don't have a little bit of knowledge of the subject field it may obviously make it difficult to translate.

P1: No, and it is very technical.

The technical nature of the scientific terms used in the test instructions, such as 'false positive rate and screen positive', was emphasised during the panel discussion. The technical nature of the test led to the detailed code regarding the need for paraphrasing $(n=14)$. Another code that featured was that there is no Northern Sotho equivalent for some of the scientific terms $(n=11)$. The technical nature of the content sometimes resulted in a lack of linguistic and content equivalence as there was no Northern Sotho word for the term, resulting in incorrect translation from the source translation to target translation. The solution posed during the panel discussion was to paraphrase the technical term to convey an accurate idea of the concept in target translation.

An example of paraphrasing was:

Source translation (English): The primary goal of the M-CHAT$\mathrm{R}^{\mathrm{TM}}$ is to maximise sensitivity, to detect as many cases of ASD as possible.

The sentence was changed to:

The main goal/objective of the M-CHAT- $\mathrm{R}^{\mathrm{TM}}$ is to detect/find as many cases of Autism as possible and translated.

Grammar and phrasing produced 12 codes that were referred to a total of 54 times. The most noticeable codes related to the back translation not being linguistically equivalent to the target translation $(n=12)$, back translation being grammatically incorrect $(n=8)$ and back translation showing incorrect subject and concord $(n=7)$. The combined themes Holistic review of test, Additional remarks and Grammar and Phrasing not only showed the high level of linguistic, construct and content equivalence that were achieved between the different translations, but also indicated the effect of technical or scientific terminology problems on the translation process. Differences in language structures between English and Northern Sotho also contributed to challenges with the back translation. The panel identified those instances where the target translation was accurately translated $(n=10)$ and indicated where differences between the target translation and back translation resulted in inaccuracies when compared with the source translation. The results and recommendations derived from the panel discussion were used in the reconciliatory test translation.

\section{Item translation and adaptation review questionnaire}

The questionnaire was completed before and after the panel discussion. Panel members answered questions according to their field of specialisation.

There was full agreement between the panel members in the written answers of the Item Translation and Adaptation Review Questionnaire regarding the Passage translation including the test introduction as well as the instructions. All the panel members agreed that the language used in the target translation does not depict individuals (e.g. toddlers at risk of ASD) in a stereotypical fashion or involve controversial topics relating to ASD. All agreed that the Item format of the test remained the same, confirming the results of the panel discussion (see Figure 1).

Minor differences in the answers of the panel members involved their views on the modification made to item structure on a grammatical level, and that the different versions convey similar content and ideas. It was indicated that some specific words may result in confusion because of multiple meanings in the target translation and that some of the content may be unfamiliar to the reader because of the technical nature.

Evidence of the value of different fields of expertise represented in the panel members became apparent in the following two questions, where zero agreement was observed: 'Are there any grammatical structures in the source language version of the item that do not have parallels in the target language?' and 'Are there any gender or other references that might make this item be cued in the target language version?' The Northern Sotho linguist (P1) was able to identify that all the grammatical structures have parallels in the target language and that gender will not have an effect in the target language. The other participants were either unsure or identified changes in structure that were evident in the back translation as they were only able to review the source translation and back translation.

One of the first language Northern Sotho SLTs (P3) and an SLT specialising in ASD (R1) reviewed the questions on General, Culture and Item format. The one question leading to a difference in opinion, related to Culture, was: 'Are there cultural differences that would have an effect on the likelihood of a response being chosen when the item is presented in the source and target language?'. The differences were identified during the panel discussion where three concepts were identified as still requiring adaptation to improve the relevance of the concepts. The additional variances included uncertainty regarding the commonality of some of the technical terms and the effect that these terms 
might have on the difficulty of the content. The Northern Sotho SLT (P3) was able to identify more of the cultural aspects and the increase in complexity of the content because of her background and understanding of Northern Sotho. The SLT specialising in ASD (R2) was again able to identify when the constructs were still equivalent even if technical language was employed.

The data obtained from the Item Translation and Adaptation Review Questionnaire confirmed the findings from the panel discussion. The convergence in the findings obtained from the expert panel discussion and the questionnaire are indicative of successful triangulation to confirm the validity of the results (Leedy \& Ormrod 2015:104). The review questionnaire allowed the participants to systematically consider the various translations. Each panel member's unique skill set influenced the rating of the translations and ensured a comprehensive panel discussion.

\section{Discussion}

As most ASD screening tests are developed in HICs and therefore culturally specific (Marlow et al. 2019:177), a double translation method was employed to adapt the M-CHAT-R $/ \mathrm{F}^{\mathrm{TM}}$ and translate the tool into Northern Sotho. By following a comprehensive three-phase process of cultural adaptation and translation, it was possible to create a culturally appropriate English version of the M-CHAT-R/FTM , as well as a Northern Sotho version of the M-CHAT-R/FT that is linguistically and conceptually equivalent. By creating a culturally sensitive screening test, the research may contribute to valuable data on atrisk rates within LMIC contexts and also result in early identification of toddlers at risk for ASD (Marlow et al. 2019:186).

Based on the answers from panel members with lived experience of the target culture, it was necessary to make four adaptations to the source translation during the Adaptation phase. A common cultural phenomenon in South Africa, including Northern Sotho culture, is the notion that it is culturally inappropriate for children to make eye contact with older people when talking to them. Children should refrain from making eye contact as a sign of respect for elders or higher status (Mncwango 2009:51). Test Item 14 was adapted to 'Does your child look in your direction or in the eye when you are talking to them?'. While the cultural reference of eye contact had to be adapted, it is a valid test item to include in a screen for ASD as atypical reaction to direct eye contact by an individual with ASD is one of the most characteristic hallmarks of the condition (Madipakkam et al. 2017:1). Both SLTs identified 'make-believe' and 'pretend play' (test Item 3) as words not familiar to the culture when referring to child play behaviour, with no conceptual equivalent in Northern Sotho. As make-believe is interpreted as an attempt to deceive someone in Northern Sotho culture, the construct was adapted to 'acting'. The lack of shared symbolic play is a valid behavioural marker for ASD. The trait is included as part of the latest diagnostic criteria for
ASD (American Psychological Association 2013), emphasising that children with ASD do not typically partake in makebelieve or pretend play.

The concepts 'Toy' and 'Equipment' were identified as 'difficult to translate'. Both toys and play equipment are cultural references, and limited exposure by children from disadvantaged communities may lead to poor comprehension of the word by parents. 'Playground equipment' in test Item 4 was adapted to a more contextually and culturally relevant concept, a tree, as children within resource-limited LMIC contexts may not be familiar with commercial playground equipment. Children from LMICs are often adversely affected by poverty, inadequate learning opportunities and lack access to equipment (Samuels, Slemming \& Balton 2012:334).

A contextually and culturally adapted English version of M-CHAT-R/FTM may be useful in South Africa as a preference for English was observed in a recent study conducted (Van der Merwe et al. 2017:5). A translation study conducted in South Africa found that younger isiZulu speaking parents preferred to answer the Parents' Evaluation of Developmental Status (PEDS) tool (Glascoe 2013) in English rather than their first language, whereas the older parents preferred the isiZulu translation (Van der Merwe et al. 2017:5). By adapting the source translation to Northern Sotho cultural references, the comprehensibility of the English version of the test was therefore enhanced for South African communities. If adaptations were not included before translation, the translation would have contained less common vocabulary that could have had a negative impact on the comprehension of specific items. Cultural adaptations to the source translation have enhanced the comprehensibility of the English version of the test not only for Northern Sotho speakers but also for other groups in Southern Africa.

The detailed codes reflected both positive and negative aspects with the review of the adaptations made in the target and back translation during the panel discussion. The review highlighted that after the initial cultural adaptation, only three constructs still required adaptation. Ortiz-Gutiérrez and Cruz-Avelar (2018:204) found that performing adaptations prior to the translation of the test enforces cultural nuance before the actual translation, this is evident as limited cultural adaptations had to be made posttranslation.

Following on the discussion of the adaptation, the most prominent positive code was that content equivalence was achieved between the target translation, back translation and the source translation. This is an indication that all the translations were indeed scrutinised to determine whether the content of the source language and the reconciliatory Northern Sotho translation were linguistically and conceptually equivalent. The high score for linguistic and concept equivalence thus implies that the target translation was a successful translation. In comparison, fewer comments were made regarding poor linguistic equivalence between 
the source and target translations. The most important reason for non-equivalence was related to technical language used in the source translation.

Technical terminology used in the source translation resulted in the identification of a significant number of detailed codes addressed during the discussion under the theme Additional remarks. This theme provides evidence that additional aspects emerged that were unrelated to the initial four themes. These detailed codes highlighted the difficulty of translating technical or scientific terms into another language that does not have a single synonymous word in the target language. According to Gouws and Prinsloo (2005:158), linguistic gaps (or, in this particular translation process, a terminological gap) occur when the speakers of both the source and target languages are familiar with a particular concept, but the target language lacks a term to designate the concept. In the current study, linguistic gaps were filled by using different term formation strategies, that is, direct borrowing, transliteration or loan translation, paraphrasing, semantic specialisation and compounding (Gauton et al. 2008:163). These strategies did not change the construct equivalence but affected the length of the test items and instructions as more words were required to paraphrase the sentence. Paraphrasing was the strategy used most often during the current translation.

According to Gauton et al. (2008), a translator is almost invariably confronted with cases of zero equivalence in any translation. Challenges related to zero equivalence are particularly prevalent when (1) the target language is an African language (Gauton et al. 2008:148) and (2) the source language veers towards the technical side, as is the case with the M-CHAT-R/F text. Translation of technical texts requires the availability of term equivalents in the target language for terms used in the source text. Apart from the fact that terminological equivalents are not always readily available to African language translators, an additional challenge is the non-standardised nature of African language terminology (Taljard 2008). Because of a lack of coordinated terminological planning evident in African languages, the translator is often confronted with multiple terms for a single concept, with no recourse to any authoritative source providing a standardised term to be used in a translation for a specific concept (Taljard 2008).

Detailed codes relating to the theme Grammar and phrasing, more specifically to the back translation, occurred frequently during the panel discussion. The purpose of the back translation was to determine the accuracy of the target translation but lead to a comprehensive discussion because of questions regarding the linguistic equivalence between the target and back translation. Grammatical errors were identified in the back translation. The differences observed in back translation typically demonstrate language interference. Language interference occurs when the surface structure of a speaker's first language is embedded in the second language (Aixela 2009:75). The absence of the definite and indefinite articles ' $a$ ', 'an' and 'the' in Northern Sotho further complicated the back translation from Northern Sotho to English. A second instance of language interference was the non-distinction between masculine and feminine pronouns in Northern Sotho, no formal (morphological) distinction exists between 'he/him' and 'she/her', thus leading to grammatical errors in the back translation. This explains the inconsistency between the target translation and back translation, indicating that the construct was still the same but the literal linguistic equivalence varied. Another aspect that had to be considered was the lack of prepositions, that is, 'to', 'from' and 'at' in Northern Sotho. The notion of prepositionality is mostly embedded in the verb stem itself or derived by means of prefixes or suffixes. This complicated the back translation to English, where prepositions and referents were required as separate words. The lack of linguistic equivalence between the target translation and back translation can thus be explained by differences in surface structure and use of paraphrasing to avoid a literal translation from the source translation to target translation. When moving past the linguistic incompatibilities, construct equivalence was still evident between the target and back translation and aligned with the aim of the double translation process of the study.

It is recommended that the current Northern Sotho version of the M-CHAT-R/F $\mathrm{F}^{\mathrm{TM}}$ should be evaluated to determine the feasibility, as well as the reliability and validity of the instrument. Investigating the language preference of South African M-CHAT-R/ $/ \mathrm{F}^{\mathrm{TM}}$, users may also indicate whether adaptation and translation of this instrument into other African languages should be considered as it may promote knowledge of ASD in SSA.

\section{Limitations of the study}

A possible limitation of the study was not including a community member as a stakeholder in the panel discussion, as the panel only included specialists and relied on the Northern Sotho specialists' cultural knowledge for the adaptation, as the Northern Sotho specialists are also considered community members.

\section{Conclusion}

The Northern Sotho translation of the M-CHAT-R/FTM is not a literal translation but a carefully constructed and culturally relevant translation of the source language which included the paraphrasing of technical terms to promote linguistic equivalence. While the double translation process highlighted some instances of differences between the target translation and back translation, it also contributed to an adapted and translated test that is equivalent and not only a literal translation of the source text. Including various specialists from different fields in the panel discussion and self-completed questionnaireallowed for a multidimensional, transdisciplinary review and interpretation of the target translation. The holistic review resulted in triangulated results supporting the translation of a culturally relevant screening test. The preliminary English adaptation, as well as the Northern Sotho 
translation of the M-CHAT-R/FTM can now be confirmed by gathering empirical evidence of reliability and validity.

\section{Acknowledgements Competing interests}

The authors declare that they have no financial or personal relationships that may have inappropriately influenced them in writing this article.

\section{Authors' contributions}

C.V. was supervised by A.K. and J.v.d.L. M.L. and E.T. supported with the interpretation and supervision of the focus group results and writing of the article.

\section{Ethical considerations}

The research was approved by the Research Ethics Committee of Humanities, University of Pretoria. The research was additionally approved by the Health Sciences Research and Ethics Committee, University of Pretoria. Number: HUM041/0919

\section{Funding information}

No specific grant from any funding agency in the public, commercial or not-for-profit sectors supported this research.

\section{Data availability}

Data will be made available by the principal researcher on request. It is available in electronic format.

\section{Disclaimer}

The views and opinions expressed in this article are those of the authors and do not necessarily reflect the official policy or position of any affiliated agency of the authors, and the publisher/s.

\section{References}

Abubakar, A., Holding, P., Van Baar, A., Newtown, C.R.J.C. \& Van de Vijver, F.J.R., 2008 'Monitoring psychomotor development in a resource-limited setting: An evaluation of the Kilifi developmental inventory', Annals of Tropical Paediatrics evaluation of the Kilifi developmental inventory', Annals of
28(3), 217-226. https://doi.org/10.1179/146532808X335679

Aixela, F.J., 2009, 'An overview of interference in scientific and technical translation', The Journal of Specialised Translation (11), 75-87.

American Psychological Association, 2013, Diagnostic and statistical manual of mental disorders (DSM-5), American Psychiatric Publishing, Arlington, TX.

Beacham, C., Reid, M., Bradshaw, J., Lambha, M., Evans, L., Gillespie, S. et al., 2018 'Screening for autism spectrum disorder: Profiles of children who are missed', Journal of Developmental and Behavioral Pediatrics 39(9), 673-682. https://doi. org/10.1097/DBP.0000000000000607

Centres for Disease Control and Prevention, 2012, 'Prevalence of autism spectrum disorder - Autism and developmental disabilities monitoring network, 14 Sites, United States, 2008', Morbidity and Mortality Weekly Report 61(3), 1-19.

Centres for Disease Control and Prevention, 2014, 'Prevalence of autism spectrum disorder among children aged 8 years', Morbidity and Mortality Weekly Report Surveillance Summaries 63(2), 1-21.

Fisher, V.J., Morris, J. \& Martines, J., 2014, 'Developmental screening tools: Feasibility of use at primary healthcare level in low- and middle-income settings', Journal of Health Population Nutrition 32(2), 314-326.

Franz, L., Chambers, N., Von Isenburg, M. \& De Vries, P.J., 2017, 'Autism spectrum disorder in sub-Saharan Africa: A comprehensive scoping review', Autism Research 10(5), 723-749. https://doi.org/10.1002/aur.1766
Fyvie, L., Anderson, J., Kruger, C.J., Le Roux, M. \& Van der Linde, J., 2016, 'The outcome of a developmental screening tool (PEDS) in English and Northern Sotho: A comparative study', Language Matters 47(3), 415-426. https://doi.org/10.1080/1 comparative study', Lang

Gauton, R., Taljard, E., Mabasa, T. \& Netshitomboni, L., 2008, 'Translating technical (LSP) texts into the official South African languages: A corpus-based investigation of translators' strategies', Language Matters 39(2), 148-180. https://doi. org/10.1080/10228190802579569

Glascoe, F.P., 2013, Collaborating with parents: Using Parents' Evaluation of Developmental Status to detect and address developmental and behavioural problems, viewed n.d., from https://www.PEDStest.com.

Gouws, R.H. \& Prinsloo, D.J., 2005, Principles and practice of South African lexicography, African Sun Media, Stellenbosch.

Guthrie, W., Wallis, K., Bennett, A., Brooks, E., Dudley, J., Gerdes, M. et al., 2019, 'Accuracy of autism screening in a large pediatric network', Pediatrics 144(4), e20183963. https://doi.org/10.1542/peds.2018-3963

Hambleton, R.K. \& Zenisky, A.L., 2011, 'Translating and adapting tests for crosscultural assessment', in D. Mutsamoto \& F.J.R. Van de Vijver (eds.), Cross-cultural research methods in psychology, pp. 46-74, Cambridge University Press, New York, NY.

Hyman, S.L., Levy, S.E., Myers, S.M. \& AAP Council on Children with Disabilities, Section on Developmental, and Behavioural Pediatrics, 2020, 'Identification,
evaluation, and management of children with autism spectrum disorder', evaluation, and management of children with autism spectrum
Pediatrics 124(1), e20193447. https://doi.org/10.1542/peds.2019-3447

International Test Commission (ITC), 2016, ITC guidelines for translation and adapting tests: Second edition, viewed 12 October 2019, from https://www.intestcom.org/ tests: Second edition, viewed 12 October
files/guideline_test_adaptation_2ed.pdf.

Kautzky, K. \& Tollman, S.M., 2008, 'A perspective on primary health care in South Africa', in South African Health Review, pp. 17-30, Health Systems Trust, University of the Witwatersrand.

Leedy, P.D. \& Ormrod, J.E., 2015, Practical research: Planning and design, Pearson, London.

Madipakkam, A.P., Rothkirch, M., Dziobek, I. \& Sterzer, P., 2017, 'Unconscious avoidance of eye contact in autism spectrum disorder', Scientific Reports 7, 13378. https://doi.org/10.1038/s41598-017-13945-5

Marlow, M., Servili, C. \& Tomlinson, M., 2019, 'A review of screening tools for the identification of autism spectrum disorder and developmental delay in infants and young children: Recommendations for use in low- and middle-income countries', Autism Research 12(2), 176-199. https://doi.org/10.1002/aur.2033

Mncwango, E.M., 2009, 'Language and the current challenges in the South African schools system', Inkanyiso: Journal of Humanities and Social Sciences 1(1), 51-54. https://doi.org/10.4314/ijhss.v1i1.62110

Morelli, D.L., Pati, S., Butler, A., Blum, N.J., Gerdes, M., Pinto-Martin, J. et al., 2014 'Challenges to implementation of developmental screening in urban primary care: A mixed methods study', Pediatrics 14(16), 1-11. https://doi.org/10.1186/14712431-14-16

Ortiz-Gutiérrez, S. \& Cruz-Avelar, A., 2018, 'Translation and cross-cultural adaptation of health assessment tools', Actas Dermosifiliograficas 109(3), 202-206. https:// doi.org/10.1016/j.ad.2017.09.012

Rahman, A.Z., Waheed, I.W. \& Hussain, N., 2003, 'Translation and cultural adaptation of health questionnaires', The Journal of the Pakistan Medical Association 53(4), 142-147.

Robins, D.L., Casagrande, K., Barton, M., Chen, C.A., Mumont-Mathieu, T. \& Fein D., 2014, 'Validation of the modified checklist for autism in toddlers, revised with follow-up', Pediatrics 133(1), 37-45. https://doi.org/10.1542/peds.2013-1813

Robins, D.L., Fein, D. \& Barton, M., 2018, 'Modified checklist for autism in toddlers, revised, with follow-up (M-CHAT-R/F)', Self-published. https://doi.org/10.1007/9783-319-57111-9_1569

Saldaña, J., 2016, The coding manual for qualitative researchers, 3rd edn., Sage, Thousand Oaks, CA.

Samuels, A., Slemming, W. \& Balton, S., 2012, 'Early childhood intervention in South Africa in relation to the developmental systems model', Infants and Young Children 25(4), 334-345. https://doi.org/10.1097/IYC.0b013e3182673e12

Schurink, W., Fouché, C.B. \& De Vos, A.S., 2015, 'Qualitative data analysis and interpretation', in A.S. De Vos, H. Strydom, C.B. Fouché \& C.S.L. Delport (eds.), Research at grass roots, pp. 397-423, Van Schaik Publishers, Cape Town.

Soto, S., Jacobstein, K.L.D., Biel, M., Migdal, T. \& Anthony, B.J., 2015, 'A review of cultural adaptations of screening toolscreening? tests for autism spectrum disorders', Autism 19(6), 646-661. https://doi.org/10.1177/1362361314 disorders
541012

StatsSA, 2019, City of Tshwane: People, viewed 11, July 2019, from http://www. statssa.gov.za/?page_id=993\&id=city-of-tshwane-municipality.

Stewart, L.A. \& Lee, C.I., 2017, 'Screening for autism spectrum disorder in low- and middle-income countries: A systematic review', Autism 21(5), 572-539. https:// doi.org/10.1177/1362361316677025

Sutton, J. \& Austin, Z., 2015, 'Qualitative research: Data collection, analysis, and management', Canadian Journal of Hospital Pharmacy 68(3), 226-231. https:// doi.org/10.4212/cjhp.v68i3.1456

Taljard, E., 2008, 'Terminology practice in a non-standardised environment: A case study', paper presented at EURALEX International Congress, Barcelona, Spain, July 15-19.

Van der Merwe, M., Cilliers, M., Maré, C., Van der Linde, J. \& Le Roux, M., 2017, 'Evaluation of a Zulu translation of the parents' evaluation of developmental
status', African Journal of Primary Health Care and Family Medicine 9(1), 1-6. https://doi.org/10.4102/phcfm.v9i1.1365 
World Health Organization, 2005, 'Disability, including prevention, management and rehabilitation. Agenda item 13.13', in Fifty-eight world health assembly, pp. 97-100, World Health Organization, Geneva.

World Health Organization, 2013, Autism spectrum disorders \& other developmental disorders. From raising awareness to building capacity, Geneva, viewed 15 August 2019, from http://apps.who int/iris/bitstream/10665/103312/1/9789241506618 ens.pdf.
World Health Organization, 2019, 'Process of translation and adaptation of instruments', viewed 15 June 2019, from https://www.who.int/substance abuse/ research tools/translation/en/.

Zwaigenbaum, L., Bauman, M.L., Stone, W.L., Yirmiya, N., Estes, A., Hansen, R.L. et al. 2015, 'Early identification of autism spectrum disorder: Recommendations for practice and research', Pediatrics 136, S-S40. 